\title{
The Study based on the Fuzzy Analytic Hierarchy Process and Data Envelopment Analysis Evaluation Model of Modern Sports Teaching
}

\author{
Xinhong Yan ${ }^{1}$, Zhenchao $\mathrm{Wu}^{1}$, Bo Hu${ }^{2}$ \\ ${ }^{1}$ Department of Sports, Shijiazhuang University of Economics, Shijiazhuang, China \\ yan_xinhong@126.com \\ ${ }^{2}$ Department of Sports, Shijiazhuang TIEDAO University SIFANG College, Shijiazhuang, China
}

Keywords: fuzzy theory; analytical hierarchy process; physical education; modernization; data envelopment analysis

\begin{abstract}
With the wide application of the modern technology of the multi-media computer, the traditional physical education method is facing more and more serious challenges and the defects of traditional physical education method emerge quickly. Thus, new modern educational means becomes nonnegligible in the role of education. Some serious problems and deficiencies are existed in the traditional evaluation system of the teaching physical education, which mainly adopts the single evaluation system. At the same time, the modern teaching is advocated in the society. The past evaluation system can not reflect the aims and requirements of modern teaching. Therefore, on the basis of the combination of the educational law of higher physical education, the two more scientific comprehensive evaluation system of physical education which conforms to modern teaching requirements are established in the paper from the teaching and education quality.
\end{abstract}

\section{Introduction}

With the development of the times and the progress of science and technology, modernization has merged into people's life. In general, the modernization refers to all the global process of underdeveloped countries with the social and cultural change in order to gain some advantages of the developed countries. If the education fulfills the prospect of the socialist modernization, it must achieve the modernization. However, the school physical education is an important part of the education. Modernization of education has close relations with sports modernization. Thus, the higher physical education modernization is the requirements of social and educational progress.

At present, the education and teaching concept which the college physical education mainly adopts is Soviet physical education teaching concept. We have introduced a lot of foreign advanced sports teaching methods since the 1980s, but in essence, a lot of methods are unfit for our national conditions. In other words, the educational teaching method with Chinese characteristic has not formed. Specifically speaking, there are still a lot of defects in the sports teaching concept, course construction and teaching methods.

Obviously, the traditional sports teaching methods can not meet the demands of the society. At the same time, the evaluation system which measures the physical education will create a great deal of defects and problems. The traditional evaluation system mainly adopts a singe evaluation system. It can not reflect the aims and requirements of modern teaching.

So the paper mainly researches the sports education evaluation model to meet the demand of modernization. Of course, the modern sports education evaluation has a wide-ranging scope. The paper establishes the comprehensive evaluation model from the two aspects of teaching quality and physical education evaluation under quality-oriented education.

Sports teaching quality evaluation usually refers to the accurate judgment based on all the factors in the teaching process and the comprehensive results. It mainly includes the teacher's teaching evaluation and students' learning evaluation. The teacher's teaching evaluation mainly takes the teacher's teaching mode as the evaluation object. The students' learning evaluation mainly takes the students' final grade as the evaluation object. The past teaching quality evaluation only paid attention to the unilateral evaluation of teacher's teaching method and students' final grade. It can not embody the inner dialectical relationship between teaching and learning. Thus, teaching efficiency is introduced, the deficiency of the old system and the new comprehensive evaluation system is established on the basis of the past evaluation system. 
The sports education under the quality education is a hot topic in the research area at present. The paper mainly studies the evaluation system of physical education under the quality education. The traditional course evaluation system mainly takes students' achievements as the standard. Furthermore, the evaluation of physical education is more difficult than that of other courses. So it is imperative to improve the old evaluation system. The paper mainly adopts three kinds of evaluation indexes of society, biology and psychology to establish the evaluation system and the corresponding evaluation model.

\section{The teaching quality fuzzy analytic hierarchy process evaluation model}

The model combines qualitative description and quantitative description, including three aspects: students' evaluation of their teachers, teacher's evaluation of students and teaching efficiency.

First of all, the paper makes the students' questionnaires survey towards their teacher, then the questionnaires of the two hundreds college students of the city are chosen at random. On that basis, we obtained the students' form of evaluation towards teacher. The form of evaluation includes six kinds of first level indexes and fourteen kinds of secondary level indexes.

The paper sets up five kinds of ratings: excellent, good, qualified, unqualified and poor. The analytical hierarchy process is used to determine the weight of indexes and the rating score, see table 1.

Using the fuzzy comprehensive evaluation method, and then let $W_{i}$ be synthetic weight and $V_{j}$ be ratings, the membership degree distribution $B_{j}=\sum W_{i} R_{i j}$ is obtained, $\sum W_{i}=1$ and $R_{i j}$ is membership degree; Then the students' evaluation value towards teacher is $G_{G_{T}}=\sum B_{j} V_{j}^{T}, V_{j}^{T}$ is transposed matrix of rating score. The data in the table 1 is substituted in the fuzzy comprehensive evaluation method, $B_{j}=(0.146,0.509,0.31,0.02,0.01)$ and $G_{T}=0.764$ are obtained.

TABLE I.

EVALUATION SHEET

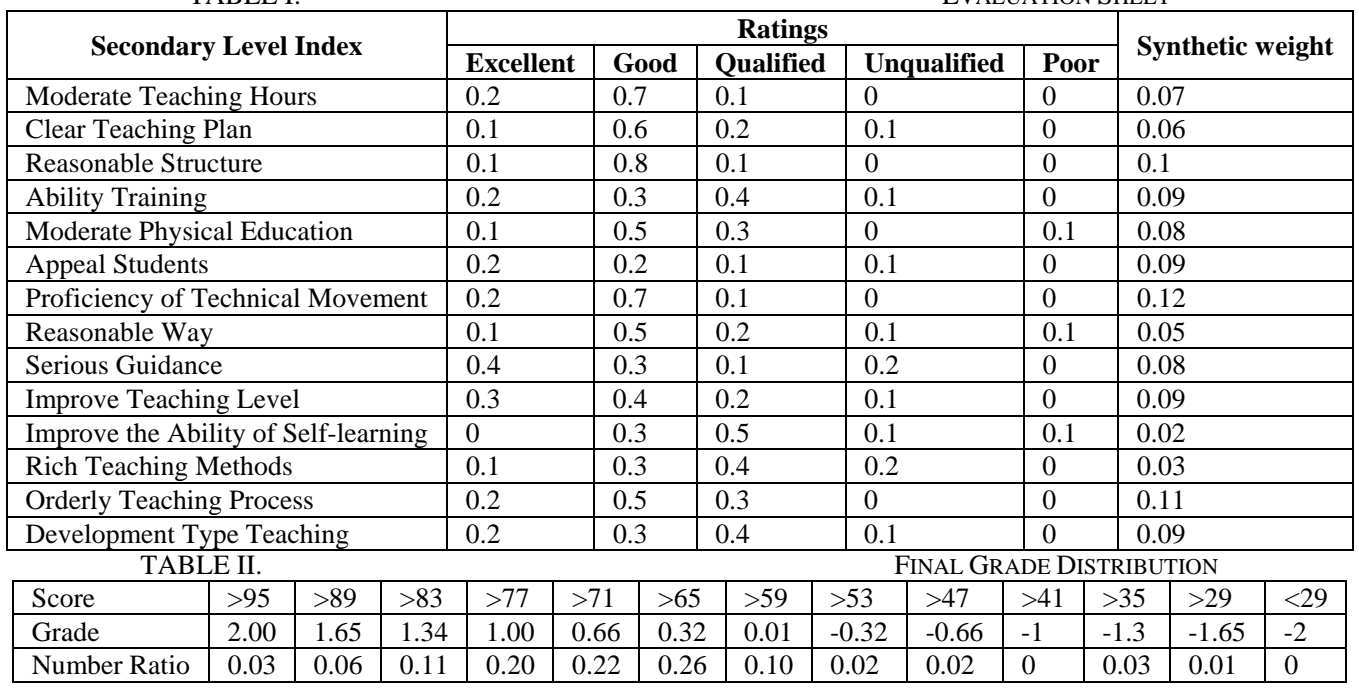

Secondly, calculate the score of teachers' evaluation towards students. The paper establishes the structure of students' final results and then establishes the thirteen kinds of ratings, see table 2.

The formula ${ }_{G_{s}}=\sum R_{j} V_{j}^{T}$ is used to get the teachers' evaluation score towards students is $G_{s}=0.682$, and $V_{j}^{T}$ is transposed matrix of thirteen kinds of rating score and $R_{j}$ is number ratio of thirteen assessment levels.

Finally, establish the teaching efficiency model. Teaching efficiency measures the quality level of teaching and learning activities, reflecting the functional relationship of quality level of learning and teaching activities. Let $H_{(T)}$ express teachers' lecture efficiency, and if $-2<G_{T}<2, \quad 0 \leq G_{s}<2$, then

If $G_{s}<0$, then

$$
H_{(T)}=\ln \left(1+\frac{G_{s} \sqrt{\left(2-G_{T}\right)\left(2-G_{s}\right)}}{\left(2-G_{T}\right)^{2}}\right)
$$

$$
H_{(T)}=\ln \left(1+\frac{G_{s} \sqrt{\left(2-G_{T}\right)\left(2-G_{s}\right)}}{\left(2-G_{s}\right)^{2}}\right)
$$


From the above two formula, we obtain that when ${ }^{G_{T}}=2$, the students' lecture evaluation towards teacher is full mark; when $G_{T}=-2$, the students' lecture evaluation towards teacher is zero. At the same time, the two formulas are used to get ${ }^{H_{(T)}}$, see table 3 .

\begin{tabular}{|l|l|l|l|l|l|l|l|l|l|l|l|l|l|}
\hline$H_{(T)}$ & -1 & 0 & 0.2 & 0.4 & 0.6 & 0.8 & 1 & 1.2 & 1.4 & 1.5 & 1.6 & 1.8 & 1.9 \\
\hline-1 & -0.4 & 0 & 0.05 & 0.1 & 0.13 & 0.16 & 0.18 & 0.19 & 0.19 & 0.18 & 0.17 & 0.14 & 0.1 \\
\hline 0 & -0.31 & 0 & 0.09 & 0.16 & 0.22 & 0.27 & 0.3 & 0.31 & 0.32 & 0.32 & 0.3 & 0.25 & 0.2 \\
\hline 0.2 & -0.28 & 0 & 0.11 & 0.19 & 0.26 & 0.31 & 0.35 & 0.37 & 0.45 & 0.36 & 0.35 & 0.29 & 0.22 \\
\hline 0.4 & -0.27 & 0 & 0.12 & 0.22 & 0.3 & 0.36 & 0.4 & 0.41 & 0.43 & 0.42 & 0.41 & 0.33 & 0.3 \\
\hline 0.6 & -0.25 & 0 & 0.16 & 0.27 & 0.36 & 0.42 & 0.47 & 0.5 & 0.51 & 0.49 & 0.48 & 0.4 & 0.31 \\
\hline 0.8 & -0.23 & 0 & 0.18 & 0.33 & 0.43 & 0.51 & 0.57 & 0.6 & 0.61 & 0.59 & 0.57 & 0.48 & 0.38 \\
\hline 1 & -0.2 & 0 & 0.24 & 0.41 & 0.54 & 0.63 & 0.69 & 0.73 & 0.72 & 0.73 & 0.7 & 0.59 & 0.47 \\
\hline 1.2 & -0.18 & 0 & 0.32 & 0.53 & 0.69 & 0.8 & 0.87 & 0.92 & 0.92 & 0.91 & 0.88 & 0.75 & 0.61 \\
\hline 1.4 & -0.16 & 0 & 0.45 & 0.74 & 0.93 & 1.06 & 1.15 & 1.2 & 1.2 & 1.19 & 1.16 & 0.99 & 0.81 \\
\hline 1.5 & -0.14 & 0 & 0.56 & 0.89 & 1.1 & 1.25 & 1.34 & 1.38 & 1.41 & 1.38 & 1.35 & 1.19 & 0.98 \\
\hline 1.6 & -0.12 & 0 & 0.72 & 1.9 & 1.34 & 1.5 & 1.59 & 1.67 & 1.65 & 1.64 & 1.61 & 1.43 & 1.22 \\
\hline 1.8 & -0.08 & 0 & 1.39 & 1.9 & 2.2 & 2.4 & 2.45 & 2.56 & 2.57 & 2.55 & 2.51 & 2.3 & 2.05 \\
\hline 1.9 & -0.05 & 0 & 2.25 & 2.8 & 3.2 & 3.6 & 3.48 & 3.5 & 3.56 & 3.54 & 3.5 & 3.25 & 2.96 \\
\hline
\end{tabular}

According to table 3, we acquire that the relationship among the students' learning quality $G_{s}$, the teacher's lecture quality $G_{T}$ and the teaching efficiency $H_{(T)}$ is not simple linear relationship, but interrelate and correlate relations function. Further, we set five kinds of evaluation criterion of $H_{(T)}: H_{(T)}<0$ expresses the poor lecture state; $H_{(T)}=0$ expresses the general lecture state; $0<H_{(T)} \leq 0.6$ expresses medium lecture state; $0.6<H_{(T)} \leq 1$ expresses good lecture state; $H_{(T)}>1$ expresses the excellent lecture state.

The obtained results $G_{T}=0.764$ and $G_{s}=0.682$, and then ${ }_{H_{(T)}}=\ln \left(1+\frac{G_{s} \sqrt{\left(2-G_{T}\right)\left(2-G_{s}\right)}}{\left(2-G_{T}\right)^{2}}\right)$ is combined to obtain $H_{(T)}=0.45$. So the evaluation result we obtained is state of teaching and learning is medium according to the model.

\section{Data envelopment analysis evaluation model of physical education under quality education}

Data envelopment analysis uses the mathematical programming model (include linear programming and multi-objective programming). Evaluation has multiple inputs and especially multiple outputs relative validity of decision making unit. Its advantage lies in the accuracy of the objective data, but it is difficult to find out the accurate data of index factor, so it has fuzziness. In this paper, the accuracy of data envelopment analysis and the fuzziness of fuzzy comprehensive evaluation are complemented with each other to obtain the data envelopment analysis evaluation mode, and it is divided into three steps: the first step, calculate the non-quantitative index weight fuzzily; the second step, the data envelopment analysis is used to calculate the quantitative index weight accurately and make the results fuzzification; the third step, the final evaluation results is obtained through fuzzy comprehensive evaluation of the above results.

If the model has $m$ evaluation unit, $(c+d)$ evaluation indexes, $c$ quantitative indicators and $d$ nonquantitative indexes.

A. The non-quantitative weight fuzzy operation

If $C=\left(c_{1}, c_{2}, \cdots, c_{q}\right)$ is factor set, $V=\left(v_{0}, v_{1}, \cdots, v_{p-1}\right)$ is comment set, then comprehensive evaluation matrix is

a, $j=1,2, \cdots, m$,

$A_{j}=\left(a_{j 1}, a_{j 2}, \cdots, a_{j q}\right)$ is weight array. So, the ${ }^{j}$ non-quantitative index weight of decision making unit fuzzy operation is

$$
B_{j}=A_{j} R_{j}=\left(a_{j 1}, a_{j 2}, \cdots, a_{j q}\right)\left[\begin{array}{cccc}
r_{j 10} & r_{j 11} & \cdots & r_{j 1(p-1)} \\
r_{j 20} & r_{j 21} & \cdots & r_{j 2(p-1)} \\
\cdots & \cdots & \cdots & \cdots \\
r_{j q 0} & r_{j q 1} & \cdots & r_{j q(p-1)}
\end{array}\right]=\left(b_{j 1}, b_{j 2}, \cdots, b_{j p}\right)
$$

B. The quantitative data envelopment of weight calculation

Assume $X_{j}=\left(x_{1 j}, x_{2 j}, \cdots, x_{n j}\right)^{T}, Y_{j}=\left(y_{1 j}, y_{2 j}, \cdots, y_{s j}\right)^{T}$ is the input and output vector of the $i$ th evaluation unit $D M U_{i}(1 \leq i \leq m)$, and $j=1,2, \cdots, m$, each vector coordinate is positive. If 
$v=\left(v_{1}, v_{2}, \cdots, v_{n}\right)^{T}, u=\left(u_{1}, u_{2}, \cdots, u_{s}\right)^{T}$ is used to express the input and output of the weight vector, and Charnes - Cooper is used to converse to obtain the linear programming model:

$$
\left\{\begin{array}{c}
\max \mu^{T} Y_{j 0} \\
\text { s.t. } \quad \omega^{T} X_{j}-\mu^{T} Y_{j} \geq 0, j=1,2, \cdots, m \\
\omega^{T} X_{j 0}=1 \\
\omega \geq 0, \mu \geq 0
\end{array}\right.
$$

The data is substituted to the model, and the optimal solution $B_{j}{ }^{\prime}$ is obtained, that is the precise calculation quantitative index weight.

The data is obtained by the data envelopment analysis method is more objective and more convincing, but not possess such membership form of perceptual cognition and fuzzy comprehensive evaluation as “ excellent, good, qualified and unqualified”. So, the membership functions are applied to make the result fuzzification.

The data envelopment analysis operation results is regarded as the degree of membership of comment set $V=\left(v_{0}, v_{1}, \cdots, v_{p-1}\right)$, and assume $r=\left(r_{0}, r_{1}, \cdots, r_{p-1}\right)$ is membership, then

$$
r_{j}=\left\{\begin{array}{l}
\frac{x-(j-1) \frac{1}{p-1}}{\frac{1}{p-1}},(j-1) \frac{1}{p-1} \leq x<j \frac{1}{p-1} \\
\frac{(j+1) \frac{1}{p-1}-x}{\frac{1}{p-1}}, j \frac{1}{p-1} \leq x<(j+1) \frac{1}{p-1} \\
0
\end{array}, \quad r_{j} \in[0,1], j=0,1, \cdots, p-1 .\right.
$$

$B_{j}^{\prime}$ is substituted into the above formula, the membership is $B_{j}=\left(b_{j 1}, b_{j 2}, \cdots, b_{j p}\right)$.

\section{Comprehensive Evaluation}

The above results are carried on the comprehensive evaluation. The comprehensive evaluation matrix is $R_{j}=\left[\begin{array}{c}B_{j 1} \\ B_{j 2} \\ \cdots \\ B_{j k}\end{array}\right], j=1,2, \cdots, m$, and $k$ is the item number of all indexes (non-quantitative and quantitative). Assume $A_{j}=\left(a_{j 1}, a_{j 2}, \cdots, a_{j k}\right), j=1,2, \cdots, m \quad$ is weight, and then

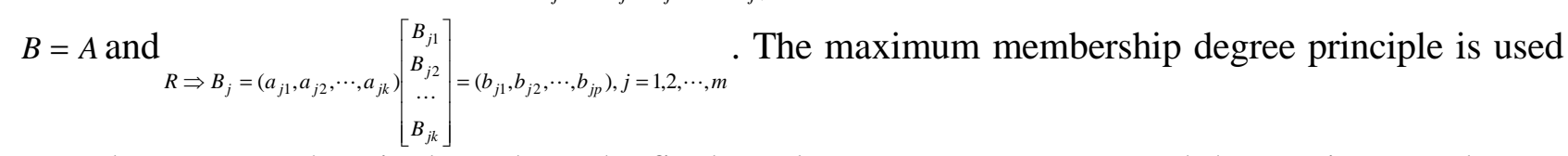
to evaluate comprehensively and get the final results $B_{j}=\left(b_{j 1}, b_{j 2}, \cdots, b_{j p}\right)$, and the maximum value $b_{j i}$ is corresponding to $v_{i}$ of $\left(v_{0}, v_{1}, \cdots, v_{p-1}\right)$.

Let $U=\left\{U_{A}, U_{B}, U_{C}\right\}$ express the evaluation set and $U_{A}=\left\{U_{A 1}, U_{A 2}, U_{A 3}, U_{A 4}\right\}=\{$ knowledge, physical quality, ability and technology $\}$ express biological factors, $U_{B}=\left\{U_{B 1}, U_{B 2}, U_{B 3}, U_{B 4}\right\}=\{$ intelligence, endurance, self-control and perception $\}$ express psychological factors, $U_{C}=\left\{U_{C 1}, U_{C 2}, U_{C 3}, U_{C 4}\right\}=\{$ sports concept, sports interest, adaptive capacity, physical quality $\}$ express social factors. Then first class index has three kinds, the secondary class index has sixteen kinds.

Let $m=\left\{m_{A}, m_{B}, m_{C}\right\}$ express weight distribution set, and

$$
m_{A}=\left\{m_{A 1}, m_{A 2}, m_{A 3}, m_{A 4}\right\}=\{0.3,0.2,0.2,0.1\} \quad, \quad m_{B}=\left\{m_{B 1}, m_{B 2}, m_{B 3}, m_{B 4}\right\}=\{0.4,0.3,0.2,0.3\}
$$

$m_{C}=\left\{m_{C 1}, m_{C 2}, m_{C 3}, m_{C 4}\right\}=\{0.3,0.2,0.3,0.2\} . V=\{$ excellent, good, medium, qualified and poor $\}$ is rating.

If we evaluate a student's psychological factors, the evaluation of four secondary indexes intelligence, endurance, self-control and perception are $\{0.2,0.3,0.3,0.15,0.05\} 、\{0.15,0.4,0.3$, $0.15,0\} 、\{0.3,0.35,0.3,0.05,0\}$ and $\{0.25,0.3,0.2,0.15,0.1\}$, then the evaluation matrix is obtained:

$$
R=\left(\begin{array}{ccccc}
0.2 & 0.3 & 0.15 & 0.3 & 0.05 \\
0.15 & 0.4 & 0.15 & 0.3 & 0 \\
0.3 & 0.35 & 0.05 & 0.3 & 0 \\
0.25 & 0.3 & 0.15 & 0.2 & 0.1
\end{array}\right)
$$

According to the weight distribution, we obtain the fuzzy matrix 


$$
R=(0.3,0.3,0.2,0.2)\left(\begin{array}{ccccc}
0.2 & 0.3 & 0.15 & 0.3 & 0.05 \\
0.15 & 0.4 & 0.15 & 0.3 & 0 \\
0.3 & 0.35 & 0.05 & 0.3 & 0 \\
0.25 & 0.3 & 0.15 & 0.2 & 0.1
\end{array}\right)=(0.2,0.3,0.3,0.15,0.1)
$$

$R=(0.19,0.29,0.28,0.15,0.09)$ is obtained through the normalization. This shows $19 \%$ of people think that the comprehensive evaluation of psychological factors is excellent, $29 \%$ think that is good, $28 \%$ think that is medium and $15 \%$ think that is qualified and $9 \%$ think that is poor.

Then we give the score to each rating: the excellent is 95 , the good is 85 , the medium is 75 , the qualified is 60 , and the poor is 50 . Thus, the acquire the score of comprehensive evaluation

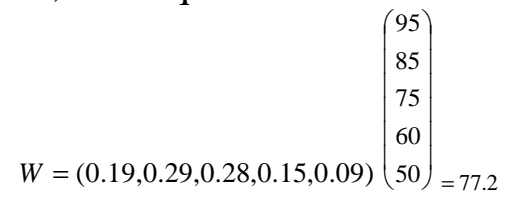

Finally, $W$ is calculated according to weight distribution, we get the score of his psychological factor is 7.72. In a similar way, the score of the other factors can be obtained.

\section{Conclusions}

With the modernization of physical education, the traditional sports teaching evaluation system has not conformed to the aims and requirements of modern teaching. The new evaluation system is established in the paper. However, modern sports education evaluation has a wide-ranging scope. The paper establishes the fuzzy process model of teaching quality and data envelopment analysis evaluation model from the two aspects of teaching quality and physical education evaluation under quality education. The two models are applied to make the scientific, objective and accurate evaluation.

\section{References}

[1] Li Qin. The Fuzzy Mathematical Model and Application of the Comprehensive Quality Evaluation of College PE Teacher. Journal of Qinghai University( Natural Science), 2008 (26) 4: 84-86.

[2] Jing Yingchuan. The Using of Mathematics Method In P. E. Teaching. Journal of Physical Education Institute of Shanxi Teachers University, 2000 (15) 2: 35-36.

[3] Sun Bingchuan, Tang Cheng. Set up Sports Teaching Evaluation System Model of University and College and Research on Excessive Member Mathematics Model. Journal of Nan Jing Institute of Physical Education (natural science), 2006 (20) 1: 69-72.

[4] Tan Pingping, Zhou Tiejun, Li Yanling, Zhou Jiamin, Tang Yuenian. Study of a Model of Sports Social Evaluation. Journal of Beijing University of Physical Education, 2005 (28) 1: 2224.

[5] Wu Xiaoqiang, Zhang Aiping, Xue Liucheng, Wang Xinguo, YuanYufeng. Research on Setting up Model for Quality Oriented Education Evalu-ation in Physical Education of Colleges and Universities in China. Journal of Anhui Sports Science, 2003 (24) 2: 68-70.

[6] Jiao Baocong, Zhao Yihuan, Dong liming. Education Informatizaton Performance Evaluation Model Based on Data Envelopment Analysis. E-education Research, 2007, 4: 38-61.

[7] Tang Lijun, Zhang Jianbin, Ji liu. College sports Modernization and PE Teachers' Quality Analysis. Spots culture guide, 2010, 6: 81-82.

[8] Liu Zhiqun, Wu Di. The Applicative Research of AHP in Sport Teaching's Quality Evaluation. Journal of Lang Fang Teachers College( Natural Science), 2010 (10) 6: 134-136.

[9] He Jieming. Application of Fuzzy Matter Element Clustering Method in Evaluation of Sports Classroom Teaching Ability. Sports Science Research, 2012 (16) 1: 72-75.

[10] 10. Chen Xiaodan, Yang Jun, Wang Huijun. The Application of Diversified Evaluation in the Sports Teaching. Sports Research and Education, 2012 (27) 6: 56-58.

[11] Li Ying, Zhang Haizhi. The Structural Equation Analysis on College Students' Sports Scores in the Applied Research. Natural Science Journal of Harbin Normal University, 2012 (28) 1: 9092.

[12] Yang Renhong. Application of Fuzzy Comprehensive Evaluation Method in Evaluation of Physical Education teaching level. China Adult Education, 2012 11: 129-130. 